\title{
Li survey in giant stars : probing non-standard stellar physics
}

\author{
N. Lagarde ${ }^{1}$, C. Charbonnel ${ }^{1,2}$, G. Jasniewicz ${ }^{3}$, P. North ${ }^{4}$, \\ M. Shetrone ${ }^{5}$, J. Hollek ${ }^{5}$, and V. V. Smith ${ }^{6}$ \\ ${ }^{1}$ Geneva Observatory, University of Geneva, Switzerland \\ email: Nadege.Lagarde@unige.ch \\ ${ }^{2}$ CNRS UMR 5572, Université de Toulouse, France \\ ${ }^{3}$ Université de Montpellier II, CNRS/UM2 UMR 5024, France \\ ${ }^{4}$ Laboratoire d'astrophysique, Ecole Polytechnique Fédérale de Lausanne, Switzerland \\ ${ }^{5}$ University of Texas, McDonald Observatory, USA \\ ${ }^{6}$ National Optical Astronomy Observatory, Tucson, USA
}

\begin{abstract}
Lithium has long been known to be a good tracer of non-standard mixing processes occurring in stellar interiors. Here we present the results of a large survey aimed at determining the surface $\mathrm{Li}$ abundance in a sample of about 800 giant (RGB and AGB) stars with accurate Hipparcos parallaxes. We compare the observed Li behaviour with that predicted by stellar models including rotation and thermohaline mixing.
\end{abstract}

Keywords. Hydrodynamics, instabilities, Stars: abundances, evolution, rotation

\section{Introduction}

Red giant stars present surface abundance anomalies that are not explained by classical stellar evolution models and that reveal the existence of extra-mixing (i.e., non convective) processes inside stellar interiors. Thermohaline mixing (Charbonnel \& Zahn 2007) has been recently identified as the dominating process that governs the photospheric composition of low-mass bright giant stars, affecting in particular the surface Li abundances in red giants more luminous than the RGB bump. In order to test this assessment we present $\mathrm{Li}$ observations in a large sample of about 800 giant stars with Hipparcos parallaxes and compare our data for the solar-metallicity subsample with models computed with the evolutionary code STAREVOL and including thermohaline mixing and rotation-induced processes (see Charbonnel \& Lagarde, this volume).

Observations were carried out with: (1) Standiford Cassegrain Echelle Spectrometer on the T2.1m at McDonald Observatory (2) Fiber-fed Extended Range Optical Spectrograph (FEROS) on the T1.52m at ESO ; (3) AURELIE spectrometer on the T1.52m at HauteProvence Observatory.

\section{Solar metallicity subsample}

In Fig. 1 we compare our observations with the predictions of the solar metallicity models described in Charbonnel \& Lagarde (this volume) and Lagarde \& Charbonnel (in preparation) that were computed with the code STAREVOL taking into account (1) rotation-induced processes following the formalism by Zahn (92) and Maeder \& Zahn (98), (2) thermohaline mixing as described by Charbonnel \& Zahn (07), and (3) atomic diffusion. We also use these models to determine the mass and evolutionary status of each sample star. 

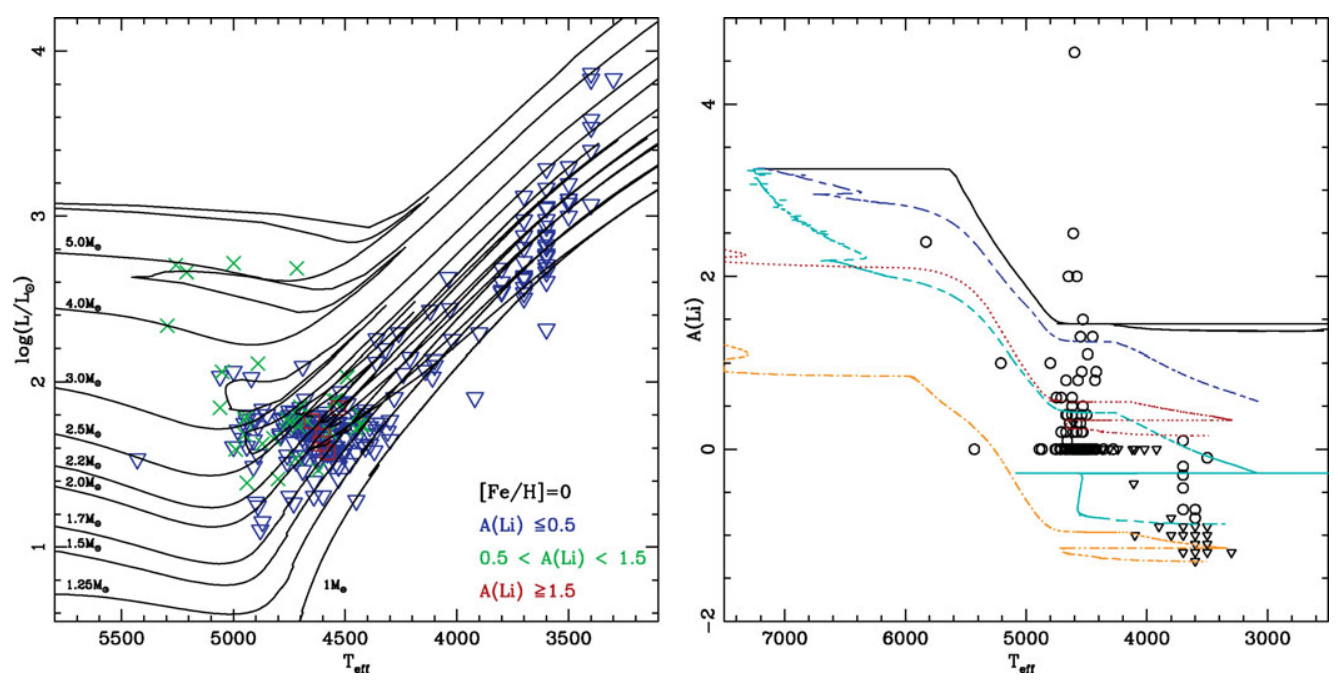

Figure 1. Comparison between models and data for the solar metallicity subsample. (Left:) HR diagram with indications on the surface Li abundance. Triangles : $A(L i) \leqslant 0.5$, square : $0.5<A(L i)<1.5$, crosses : $A(L i) \geqslant 1.5$. (Right:) Lithium as a function of Teff for stars with masses lower than $2 M_{\odot}$ (Circles and triangles for actual determinations and upper limits respectively). Solid line, short dashed - long dashed line, and dashed line represent $1.5 M_{\odot}$ stars in standard model, with thermohaline mixing and rotation $V_{Z A M S}=50 \mathrm{~km} / \mathrm{s}$ and $V_{Z A M S}=110 \mathrm{~km} / \mathrm{s}$ respectively. Dotted line and dot - short dashed line represent $2.0 M_{\odot}$ with thermohaline mixing and rotation $V_{Z A M S}=110 \mathrm{~km} / \mathrm{s}$ and $V_{Z A M S}=250 \mathrm{~km} / \mathrm{s}$ respectively.

On the main sequence and early-RGB, rotation-induced processes lead to stronger Li depletion than in the standard case, in agreement with observations, and the observed $\mathrm{Li}$ dispersion reflects dispersion in the initial rotation velocity (see also Charbonnel \& Talon 1999, Palacios et al. 2003, Smiljanic et al. 2009). After the end of the first dredgeup (Teff $\sim 4800 K)$, the Li abundance remains temporarily constant. When thermohaline mixing becomes efficient at the bump in the luminosity function (which corresponds here to Teff $\sim 4200 K$ ), the $\mathrm{Li}$ abundance is predicted to drop again in drastic manner, explaining very well the Li upper limits obtained for the brightest RGB and AGB sample stars.

\section{References}

Charbonnel, C. \& Zahn, J. P. 2007, A\& A, 467, L15

Charbonnel, C. \& Talon, S. 1999, A\&SA, 351, 635

Maeder, A. \& Zahn, J. P. 1998, A\&A, 334, 1000

Palacios, A., Talon, S., Charbonnel, C., \& Forestini, M. 2003, A\&A, 399, 603

Smiljanic, R., Pasquini, L., Charbonnel, C., \& Lagarde, N. 2009, A\&BA,in press, astro-ph 0910.4399

Zahn, J. P. 1992, A\&A, 265, 115 